\title{
Analysis of the gut microbiome of Japanese inflammatory bowel disease patients
}

\author{
Yuto Fukui ( $\nabla$ yuuto.fukui@med.toho-u.ac.jp )

\section{Hitoshi Nakajima} \\ Toho University \\ Kotaro Aoki \\ Toho University \\ Ohmori Toshihide \\ Ohmori Toshihide Gastro-Intestinal Clinic \\ Kazuhiro Tateda \\ Toho University \\ Yoshihisa Urita \\ Toho University
}

Toho university https://orcid.org/0000-0001-7318-4945

\section{Research article}

Keywords: Crohn's disease, Ulcerative colitis, Inflammatory bowel disease, Microbiome

Posted Date: July 2nd, 2019

DOl: https://doi.org/10.21203/rs.2.10838/v1

License: (c) (1) This work is licensed under a Creative Commons Attribution 4.0 International License.

Read Full License 


\section{Abstract}

Background Gut microbiome dysbiosis has been reported in patients with inflammatory bowel disease (IBD). However, gut microbiome of healthy Japanese individuals differs from that of other populations and gut microbiome of Japanese IBD patients has not been well characterized. We conducted a crosssectional study to characterize the gut microbiome of Japanese patients with Crohn's disease (CD) and ulcerative colitis (UC). Methods Two-hundred and eighty-four IBD patients (39 CD patients and $245 \mathrm{UC}$ patients) and 31 healthy participants were enrolled. Gut microbiome was analyzed by 16S rRNA amplicon sequencing using Illumina Miseq. Results Significant differences were observed among the gut microbiome of CD patients, UC patients and healthy individuals. Species richness and evenness were significantly lower in patients with active IBD than in healthy individuals. At the genus level, Bifidobacterium was most abundant genera in all three groups. Pathogenic obligate anaerobes such as Prevotella and Veillonella were increased and commensal bacteria such as Ruminococcus was decreased in IBD patients compared with healthy individuals. Conclusions CD patients and UC patients showed unique patterns of gut microbiome dysbiosis. Patients with active IBD had severe dysbiotic changes. However, characteristic features of the gut microbiome of Japanese individuals, such as high abundance of the genus Bifidobacterium, were maintained in IBD patients.

\section{Background}

Inflammatory bowel disease (IBD) is a group of chronic relapsing inflammatory disorders of the gastrointestinal tract, including both ulcerative colitis (UC) and Crohn's disease (CD) [1-3]. A number of risk factors for IBD have been identified, including ethnicity, genetics, diet and antibiotics usage, but the pathogenesis of IBD remains poorly understood $[4,1,5,2]$. Recently, the role of gut microbiota and hostmicrobiome interactions in IBD pathogenesis have been the subject of much research [6-8].

Several previous studies have reported impairment of the gut microbiota (termed dysbiosis) in IBD patients. In IBD patients, the diversity of intestinal microbiota, as reflected by species richness and evenness, appeared to decline $[7,9,8]$. The gut bacterial composition of the IBD patients also differed from that of healthy controls. At the phylum level, Proteobacteria was increased and Firmicutes was decreased in IBD patients $[10,11,8]$. Genus- and species-level analyses showed that pathogenic bacteria, including the genus Veillonella and Escherichia coli, were elevated in IBD patients [12,13]. The genera Butyricicoccus and Roseburia are thought to play a protective role against IBD by producing butyric acid, and their abundance is decreased in IBD patients $[9,6]$. These dysbiotic change may influence the development, prevention, progression and prognosis of IBD $[1,2,14]$. However, most IBD microbiome studies have been conducted in Western countries and the microbiome of Japanese IBD patients has not been well characterized.

A previous study showed that the gut microbiome of healthy Japanese individuals differed from that of other populations [15]. The gut microbiome of Japanese individuals is characterized by increased abundance of the phylum Actinobacteria, particularly the genus Bifidobacterium $[15,16]$. Functional 
analyses revealed that bacteria involved in carbohydrate metabolism were overrepresented in the intestinal tracts of Japanese individuals [15]. The prevalence of IBD is lower in Japanese than in Western populations [17], potentially due to differences in diet, genetic factors, and gut microbiome. Because the healthy gut microbiome of Japanese individuals is unique, microbiome changes that occur during IBD may differ in Japanese patients compared with individuals from other countries. Here, we conducted a cross-sectional study to characterize the gut microbiome of Japanese IBD patients.

\section{Methods}

\section{Ethics}

This cross-sectional study protocol was approved by the Ethics Committee of Toho University Omori Medical Center (Approval number: M16251). All participants provided written informed consent in accordance with the principles laid out in the Declaration of Helsinki. This study was registered at the University Hospital Medical Information Network Center (UMIN000033704).

\section{Participants and sample collection}

Patients with CD and UC were recruited from the Ohmori Toshihide Gastro-Intestinal Clinic, Saitama, Japan. Eligible patients were Japanese individuals aged 16-90 years with a well-established diagnosis of CD or UC based on clinical, endoscopic and histopathological criteria $[18,19]$. Exclusion criteria included infectious gastroenteritis, significant acute or chronic coexisting illness, antibiotic use within the last 4 weeks, and a history of colectomy. IBD patients were classified into two sub-groups (remission or active disease) according to the following criteria: (i) CD remission when CD activity index (CDAl) was < 150; (ii) active CD when CDAI was $\geq 150$; (iii) UC remission when clinical activity index (CAI) was < 4; and (iv) active UC when CAI was $\geq 4[20,21]$. Healthy Japanese volunteers were recruited from Toho University Omori Medical Center, Tokyo, Japan. Healthy participants showed no evidence of acute gastroenteritis, gastrointestinal inflammatory diseases or functional gastrointestinal disorders. In total, 284 IBD patients (39 CD patients and 245 UC patients) and 31 healthy participants were enrolled. We obtained clinical and demographic data through standardized subject interviews and review of medical records (Table 1). Study participants were instructed to collect a fecal sample within $8 \mathrm{~h}$ of their clinic visit. Collected samples were stored at $-20^{\circ} \mathrm{C}$ in the clinic, then transported to the research laboratory and stored at $-80^{\circ} \mathrm{C}$ until DNA extraction was performed.

\section{DNA extraction and sequencing}

Genomic DNA (gDNA) was extracted using a Quick-DNA Fecal/Soil Microbe Miniprep Kit (Zymo Research, Irvine, CA, USA), according to the manufacturer's protocol with minor modifications [22]. Briefly, samples were heat-lysed at $70^{\circ} \mathrm{C}$ for $10 \mathrm{~min}$ before the beating step. The mechanical bead beating step was performed using FastPrep FP120 (Thermo Savant, Carlsbad, CA, USA) for $90 \mathrm{~s}$ at speed 5.5 to enhance chemical, heat, and mechanical lysis. A blank sample was included as a control in the gDNA extraction step to assess potential contamination. 
Sequencing libraries were prepared for Illumina MiSeq sequencing (Illumina, San Diego, CA, USA). For bacterial analysis, the primer set consisting of 341F (5'-CCTACGGGNGGCWGCAG-3') and 806R (5'GACTACHVGGGTATCTAATCC-3') was used to amplify the 16S rRNA V3 and V4 regions [23]. A 16S rRNA library for the Illumina MiSeq platform was prepared as the manufacturer's protocol [24]. The library was sequenced with $2 \times 300$-bp paired-end reads on a MiSeq system using MiSeq v3 reagent kits (Illumina, San Diego, CA, USA).

\section{Sequence and statistical analyses}

MiSeq sequencing produced a total of $38,136,800$ reads with a mean output of $121,069 \pm 43,748$ reads per sample. Sequence data were submitted to DDBJ under the accession number DRA007220.

Sequencing data were processed using CLC Genomics Workbench 11.0.1 and CLC Microbial Genomics Module 3.5 (Qiagen, Redwood City, CA, USA) [25]. Overlapping paired-end reads were merged and trimmed, and chimeric reads were filtered using default parameters. The remaining reads were clustered into operational taxonomy units (OTUs) with $97 \%$ identity using the Greengenes database (version 13_5) as the reference [26]. OTUs with less than 300 reads were removed from the analysis, leaving a total of 1,107 bacterial OTUs.

Samples were rarefied to 6333 reads/sample, based on rarefaction analysis. Alpha diversity was calculated as the number of observed OTUs and Shannon entropy by CLC Genomics Workbench [27]. Beta diversity was measured as unweighted and weighted UniFrac distance based on the OTU table by CLC Genomics Workbench [28]. We used the Kruskal-Wallis test to compare alpha diversity and relative abundance values between the groups using $\operatorname{R}$ and EZR $[29,30]$. Permutational multivariate analysis of variance (PERMANOVA) was performed to compare beta diversity using CLC Genomics Workbench. Linear discriminant effect size analysis (LEfSe), which is an algorithm for identifying genomic taxa whose relative abundances differ significantly between groups [31], was used in the microbiome analysis with default parameters. $P$ values were corrected for multiple comparisons using the Bonferroni method [32]. $P$ values $<0.05$ were considered statistically significant.

\section{Results}

\section{Japanese IBD patients had altered gut microbiome}

We first assessed the alpha diversity (as reflected by the number of observed OTUs and Shannon entropy) of gut microbiome. CD patients had significantly lower species richness and evenness than UC patients and healthy participants (Fig. 1). There was no statistical difference in alpha diversity between UC patients and healthy participants (Fig. 1). Analysis of beta diversity, determined by principal coordinates analysis using unweighted and weighted UniFrac distance metrics, showed that the bacterial communities of the three clinical groups (CD, UC and healthy) were distinct from one another $(P<0.001$ and $P<0.001$, PERMANOVA) (Fig. 2 and Fig. S1). 
To determine which taxa differed between the three groups, we compared the relative abundances at different taxonomic levels. Phylum-level analysis revealed that four phyla (Firmicutes, Actinobacteria, Bacteroidetes, Proteobacteria) predominated in all three groups; the most abundant phyla were Firmicutes followed by Actinobacteria (Fig. S2). The abundance of Actinobacteria, which was shown to be predominant in the gut microbiome of Japanese individuals $[15,16]$, did not differ significantly between IBD patients and healthy participants.

A total of 48 families were detected in all three groups. The median number of observed families was lower in CD patients (23, interquartile range (IQR) 19.5-24.5) and UC patients (25, IQR 22-27) compared with healthy participants (28, IQR 24-30) (Fig. S3). At the family level, we identified the core microbiome of each group, defined as the families present in all samples [33]. The core gut microbiome consisted of 9 families in healthy participants, four families in UC patients and three families in CD patients (Fig. 3). Loss of the healthy core microbiome were observed in IBD patients; however, three families (Bifidobacteriaceae, Streptococcaceae and Veillonellaceae) were common to all three groups. Comparing the relative abundance of members of the core microbiome (Fig. 4), we identified a common perturbation in IBD patients: a significant increase of Veillonellaceae. CD patients had a significant lower abundance of Ruminococcaceae, Coriobacteriaceae and Erysipelotrichaceae than UC patients and healthy participants. UC patients had a significant lower abundance of Lachnospiraceae than healthy participants.

To identify major genus-level differences, we used LEfSe with default parameters [31]. LEfSe analysis revealed that Ruminococcus were enriched in healthy participants, Veillonella and Enterococcus were enriched in CD patients, and Megamonas, Faecalibacterium, Prevotella and Collinsella were enriched in UC patients (Fig. 5).

\section{Patients with active IBD showed severe dysbiotic changes}

We next compared the gut microbiome of patients with active disease to those of patients in remission. Among $C D$ patients and healthy individuals, patients with active CD had the lowest alpha diversity (Fig. S4). Beta diversity also differed significantly between patients with active $C D$ and healthy individuals $(P<$ 0.001 and $P<0.05$, PERMANOVA) as well as between CD patients in remission and healthy individuals ( $P$ $<0.001$ and $P<0.05$, PERMANOVA) (Fig. S5). However, no significant differences in alpha diversity (Fig. S4) or beta diversity ( $P=1$ and $P=1$, PERMANOVA) (Fig. S5) were identified between patients with active $\mathrm{CD}$ and $\mathrm{CD}$ patients in remission.

Among UC patients and healthy individuals, patients with active UC had the lowest alpha diversity both in terms of the number of observed OTUs and the Shannon index (Fig. S6). There was no significant difference of alpha diversity observed between UC patients in remission and healthy individuals (Fig. S6). Beta diversity also differed significantly between patients with active UC and healthy individuals $(P<$ 0.001 and $P<0.01$, PERMANOVA) as well as between UC patients in remission and healthy individuals ( $P$ 
$<0.001$ and $P<0.001$, PERMANOVA) (Fig. S7). However, there was no significant difference in beta diversity between the patients with active UC and UC patients in remission $(P=0.20$ and $P=1$, PERMANOVA) (Fig. S7).

\section{Discussion}

Our study identified the core gut microbiome of Japanese individuals and showed that IBD patients had dysbiotic changes compared with healthy individuals.

In agreement with previous studies analyzing the gut microbiome $[15,16]$, we observed that Japanese individulals showed high abundance of the phylum Actinobacteria, particularly the genus Bifidobacterium. Interestingly, this feature was maintained in both CD and UC patients, despite their gut microbiome dysbiosis. Bifidobacterium has been shown to have beneficial effects for human health, such as improving mucosal barrier function and reducing inflammation by producing short-chain fatty acids and vitamins, and is used in probiotics as one treatment option for IBD [34-37]. A previous Western study analyzing the treatment-naïve CD microbiome showed that Bifidobacterium abundance decreased in CD patients [7]. The high abundance of Bifidobacterium in the gut microbiome of Japanese individuals was considered as a potential factor explaining the low IBD prevalence in Japanese populations; however, in our study, this unique feature was detected not only in healthy individuals but also in IBD patients. Members of the genus Bifidobacterium can have species-dependent effects [34, 36, 37], and thus further species-level research is needed to understand the role of Bifidobacterium in the pathogenesis of IBD.

Our study showed that number of observed families significantly decreased in IBD patients with concomitant loss of the core microbiome. At the genus level, pathogenic obligate anaerobes such as Prevotella and Veillonella increased and commensal bacteria such as Ruminococcus decreased in the IBD patients, in agreement with previous reports [12, 14, 38]. Prevotella and Veillonella have been shown to promote chronic inflammation and may have an important role in chronic diseases [39, 40]. Alterations in microbiome and their metabolites are known to be involved not only in IBD pathogenesis but also in different phases of disease progression, including development of complications and extraintestinal disorders $[41,42]$.

The gut microbiome of patients with active UC, but not UC patients in remission, showed significantly decreased species richness and evenness compared with healthy individuals. Moreover, CD patients showed more severe dysbiotic changes than UC patients in terms of decreased alpha diversity. This change was more pronounced in patients with active $C D$ compared with $C D$ patients in remission. The microbial diversity of patients with active IBD might be more influenced by various factors such as increased anti-microbial proteins due to bleeding and the wash-away effect of frequent diarrhea [8]. By contrast, there was no significant difference in beta diversity between patients with active IBD and IBD patients in remission. However, the principal coordinates analysis showed that the bacterial communities of CD patients, UC patients and healthy individuals were distinct from one another. In case of difficult diagnoses, analysis of gut microbiome may contribute useful information in the future. 
This study had several limitations. First, it focused only on bacterial communities, but other organisms, such as fungi, viruses, archaea and protozoa, may also play important roles in IBD [12]. Another limitation was that we only included a few treatment-naïve IBD patients. Analyses of additional treatment-naïve IBD patients and collection of samples before and after disease onset will be needed to understand the influence of the gut microbiome on IBD.

\section{Conclusions}

We conducted a cross-sectional study to describe the gut microbiome of Japanese IBD patients and found that $C D$ patients and UC patients each showed unique patterns of gut microbiome dysbiosis. However, the characteristic features of the gut microbiome of Japanese individuals, including high Bifidobacterium abundance, were maintained in IBD patients. Disease activity affected the gut microbiome of Japanese IBD patients.

\section{Abbreviations}

CAl: clinical activity index; CD: Crohn's disease; CDAl: Crohn's disease activity index; gDNA: Genomic DNA; IBD: inflammatory bowel disease; IQR: Interquartile range; LEfSe: Linear discriminant effect size analysis; NA: Not applicable; OTU: Operational Taxonomy Unit; PERMANOVA: Permutational multivariate analysis of variance; UC: ulcerative colitis; SD: Standard deviations; WBC: White blood cell

\section{Declarations}

\section{Ethics approval and consent to participate}

This cross-sectional study protocol was approved by the Ethics Committee of Toho University Omori Medical Center (Approval number: M16251). All participants provided written informed consent in accordance with the principles laid out in the Declaration of Helsinki. This study was registered at the University Hospital Medical Information Network Center (UMIN000033704).

\section{Consent for publication}

Not applicable.

\section{Availability of data}

Sequence data were submitted to DDBJ under the accession number DRA007220.

\section{Competing interests}

The authors declare that they have no competing interests.

\section{Funding}


No funding was received to support this study.

\section{Authors' Contributions}

YF, HN, KT and YU conceived and designed the study. TO and HN performed the sampling and acquired the data. YF and KA performed laboratory analyses. YF performed data interpretation and statistical analyses. All authors performed data analysis and interpretation of the results. YF wrote this paper. All authors contributed, read and approved the final manuscript.

\section{Acknowledgements}

We thank Edanz Group (www.edanzediting.com/ac) for editing a draft of this manuscript.

\section{References}

1. Abraham C, Cho JH. Inflammatory Bowel Disease. New England Journal of Medicine. 2009;361:2066-2078.

2. Khor B, Gardet A, Xavier RJ. Genetics and pathogenesis of inflammatory bowel disease. Nature. 2011;474:307-317.

3. Taleban S, Colombel JF, Mohler MJ, et al. Inflammatory bowel disease and the elderly: a review. J Crohns Colitis. 2015;9:507-515.

4. Sakamoto N, Kono S, Wakai K, et al. Dietary risk factors for inflammatory bowel disease: a multicenter case-control study in Japan. Inflamm Bowel Dis. 2005;11:154-163.

5. Shaw SY, Blanchard JF, Bernstein CN. Association between the use of antibiotics in the first year of life and pediatric inflammatory bowel disease. The American journal of gastroenterology. 2010;105:2687-2692.

6. Morgan XC, Tickle TL, Sokol H, et al. Dysfunction of the intestinal microbiome in inflammatory bowel disease and treatment. Genome biology. 2012;13:R79.

7. Gevers D, Kugathasan S, Denson LA, et al. The treatment-naive microbiome in new-onset Crohn's disease. Cell host \& microbe. 2014;15:382-392.

8. Nishino K, Nishida A, Inoue R, et al. Analysis of endoscopic brush samples identified mucosaassociated dysbiosis in inflammatory bowel disease. Journal of gastroenterology. 2018;53:95-106.

9. Laserna-Mendieta EJ, Clooney AG, Carretero-Gomez JF, et al. Determinants of Reduced Genetic Capacity for Butyrate Synthesis by the Gut Microbiome in Crohn's Disease and Ulcerative Colitis. J Crohns Colitis. 2018;12:204-216.

10. Nagalingam NA, Lynch SV. Role of the microbiota in inflammatory bowel diseases. Inflamm Bowel Dis. 2012;18:968-984.

11. Sartor RB. The intestinal microbiota in inflammatory bowel diseases. Nestle Nutrition Institute workshop series. 2014;79:29-39. 
12. Sartor RB, Wu GD. Roles for Intestinal Bacteria, Viruses, and Fungi in Pathogenesis of Inflammatory Bowel Diseases and Therapeutic Approaches. Gastroenterology. 2017;152:327-339.e324.

13. Martinez-Medina M, Aldeguer X, Lopez-Siles M, et al. Molecular diversity of Escherichia coli in the human gut: new ecological evidence supporting the role of adherent-invasive E. coli (AIEC) in Crohn's disease. Inflamm Bowel Dis. 2009;15:872-882.

14. Sheehan D, Moran C, Shanahan F. The microbiota in inflammatory bowel disease. Journal of gastroenterology. 2015;50:495-507.

15. Nishijima S, Suda W, Oshima K, et al. The gut microbiome of healthy Japanese and its microbial and functional uniqueness. DNA research : an international journal for rapid publication of reports on genes and genomes. 2016;23:125-133.

16. Takagi $T$, Naito $Y$, Inoue $R$, et al. Differences in gut microbiota associated with age, sex, and stool consistency in healthy Japanese subjects. Journal of gastroenterology. 2019;54:53-63.

17. Asakura K, Nishiwaki Y, Inoue N, et al. Prevalence of ulcerative colitis and Crohn's disease in Japan. Journal of gastroenterology. 2009;44:659-665.

18. Kornbluth A, Sachar DB. Ulcerative colitis practice guidelines in adults: American College of Gastroenterology, Practice Parameters Committee. The American journal of gastroenterology. 2010;105:501-523; quiz 524.

19. Lichtenstein GR, Loftus EV, Isaacs KL, et al. ACG Clinical Guideline: Management of Crohn's Disease in Adults. The American journal of gastroenterology. 2018;113:481-517.

20. Best WR, Becktel JM, Singleton JW, et al. Development of a Crohn's disease activity index. National Cooperative Crohn's Disease Study. Gastroenterology. 1976;70:439-444.

21. Rachmilewitz D. Coated mesalazine (5-aminosalicylic acid) versus sulphasalazine in the treatment of active ulcerative colitis: a randomised trial. BMJ (Clinical research ed). 1989;298:82-86.

22. Leung MHY, Chan KCK, Lee PKH. Skin fungal community and its correlation with bacterial community of urban Chinese individuals. Microbiome. 2016;4:1-15.

23. Klindworth A, Pruesse E, Schweer T, et al. Evaluation of general $16 \mathrm{~S}$ ribosomal RNA gene PCR primers for classical and next-generation sequencing-based diversity studies. Nucleic Acids Res. 2013;41:e1.

24. Illumina. 16S Metagenomic Sequencing Library Preparation. http://jp.support.illumina.com/content/dam/illuminasupport/documents/documentation/chemistry_documentation/16s/16s-metagenomic-library-prepguide-15044223-b.pdf.

25. Fukui $Y$, Aoki K, Ishii $Y$, et al. The palatine tonsil bacteriome, but not the mycobiome, is altered in HIV infection. BMC microbiology. 2018;18:127.

26. DeSantis TZ, Hugenholtz P, Larsen N, et al. Greengenes, a Chimera-Checked 16S rRNA Gene Database and Workbench Compatible with ARB. Applied and environmental microbiology. 2006;72:5069-5072. 
27. Shannon CE. A mathematical theory of communication. The Bell System Technical Journal. 1948;27:379-423.

28. Lozupone $C$, Lladser ME, Knights $D$, et al. UniFrac: an effective distance metric for microbial community comparison. The ISME journal. 2011;5:169-172.

29. Team RC: A language and environment for statistical computing. R Foundation for Statistical Computing, Vienna, Austria 2017.

30. Kanda Y. Investigation of the freely available easy-to-use software 'EZR' for medical statistics. Bone marrow transplantation. 2013;48:452-458.

31. Segata N, Izard J, Waldron L, et al. Metagenomic biomarker discovery and explanation. Genome biology. 2011;12:R60.

32. Armstrong RA. When to use the Bonferroni correction. Ophthalmic \& physiological optics : the journal of the British College of Ophthalmic Opticians (Optometrists). 2014;34:502-508.

33. Turnbaugh PJ, Ley RE, Hamady M, et al. The Human Microbiome Project. Nature. 2007;449:804-810.

34. Sanders ME, Guarner F, Guerrant R, et al. An update on the use and investigation of probiotics in health and disease. Gut. 2013;62:787-796.

35. De Greef E, Vandenplas Y, Hauser B, et al. The use of probiotics in IBD and IBS. Minerva pediatrica. 2014;66:491-500.

36. Bernini LJ, Simao ANC, de Souza CHB, et al. Effect of Bifidobacterium lactis HN019 on inflammatory markers and oxidative stress in subjects with and without the metabolic syndrome. Br J Nutr. 2018:18.

37. Alard J, Peucelle V, Boutillier D, et al. New probiotic strains for inflammatory bowel disease management identified by combining in vitro and in vivo approaches. Benef Microbes. 2018;9:317331.

38. Lucke K, Miehlke S, Jacobs E, et al. Prevalence of Bacteroides and Prevotella spp. in ulcerative colitis. Journal of medical microbiology. 2006;55:617-624.

39. Segal LN, Alekseyenko AV, Clemente JC, et al. Enrichment of lung microbiome with supraglottic taxa is associated with increased pulmonary inflammation. Microbiome. 2013;1:19.

40. Larsen JM. The immune response to Prevotella bacteria in chronic inflammatory disease. Immunology. 2017;151:363-374.

41. Shanahan F, Quigley EMM. Manipulation of the Microbiota for Treatment of IBS and IBD-Challenges and Controversies. Gastroenterology. 2014;146:1554-1563.

42. Sheehan D, Shanahan F. The Gut Microbiota in Inflammatory Bowel Disease. Gastroenterology clinics of North America. 2017;46:143-154.

\section{Tables}


Table 1. Characteristics of study participants

\begin{tabular}{lccc}
\hline & CD & UC & Healthy \\
& $N=39$ & $N=245$ & $N=31$ \\
\hline Age, mean (SD), years & $39(11)$ & $48(14)$ & $45(14)$ \\
\hline Gender (male), $n$ (\%) & $31(79)$ & $139(57)$ & $31(45)$ \\
\hline Years since diagnosis, mean (SD), years & $13.2(6.8)$ & $10.6(7.8)$ & NA \\
\hline WBC, mean (SD), count/dL & $6100(1903)$ & $5866(1670)$ & NA \\
\hline Activity (active), $n$ (\%) & $7(18)$ & $98(40)$ & NA \\
\hline Type of disease, $n$ & & & \\
\hline Ileitis/ileocolitis/colitis & $9 / 17 / 13$ & NA & NA \\
\hline Proctitis/left-sided colitis/pancolitis & NA & $68 / 95 / 82$ & NA \\
\hline Medications, $n$ (\%) & & & \\
\hline 5-ASA compounds & $35(90)$ & $228(93)$ & NA \\
\hline Steroids & $26(67)$ & $13(5)$ & NA \\
\hline Immunosuppressant & $4(10)$ & $5(2)$ & NA \\
\hline Biologics & & & NA \\
\hline
\end{tabular}

Age, years since diagnosis and WBC counts were summarized by means.

\section{Figures}



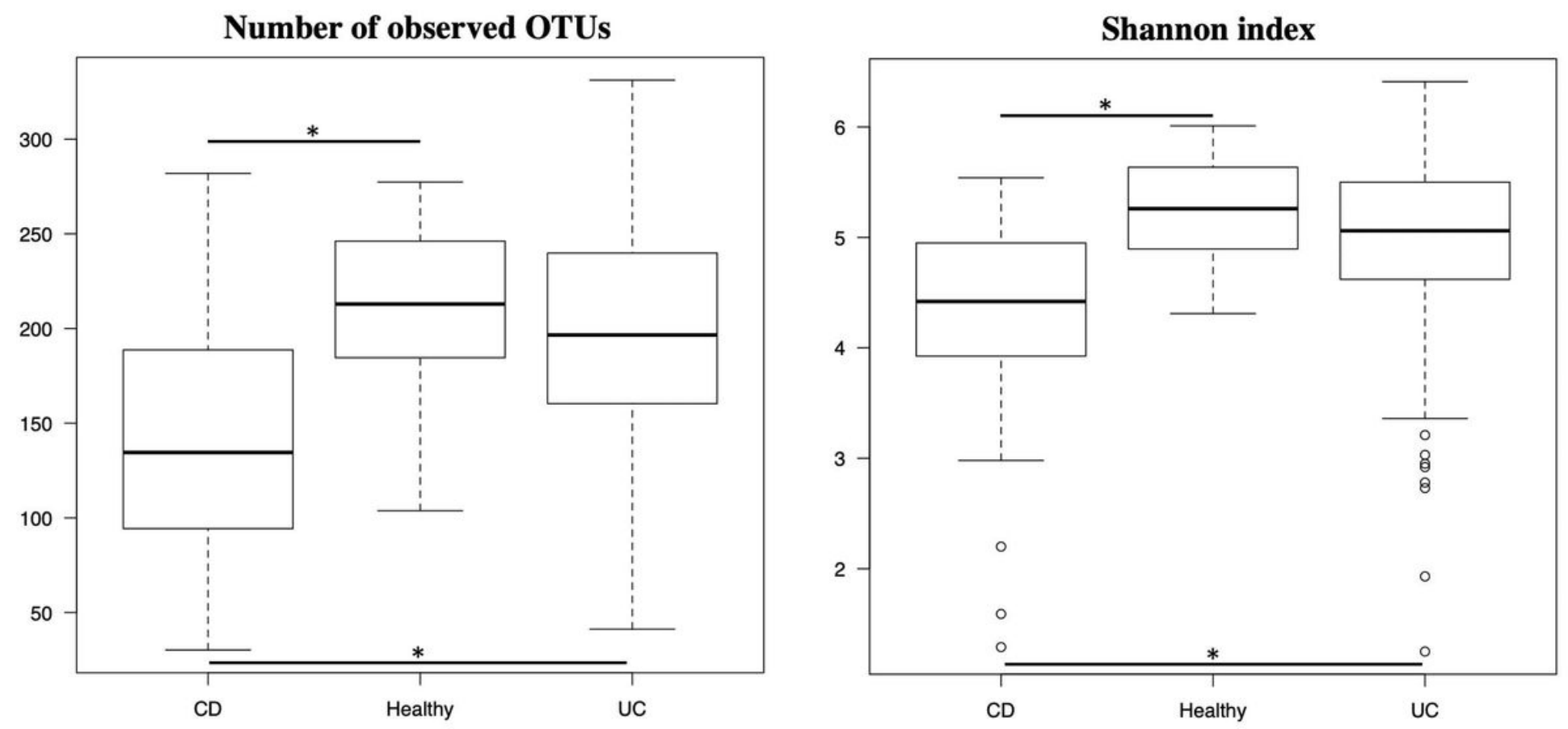

Figure 1

Alpha diversity indices of gut microbiome of CD patients, UC patients and healthy individuals. ${ }^{*} p<0.001$, the Kruskal-Wallis test. 


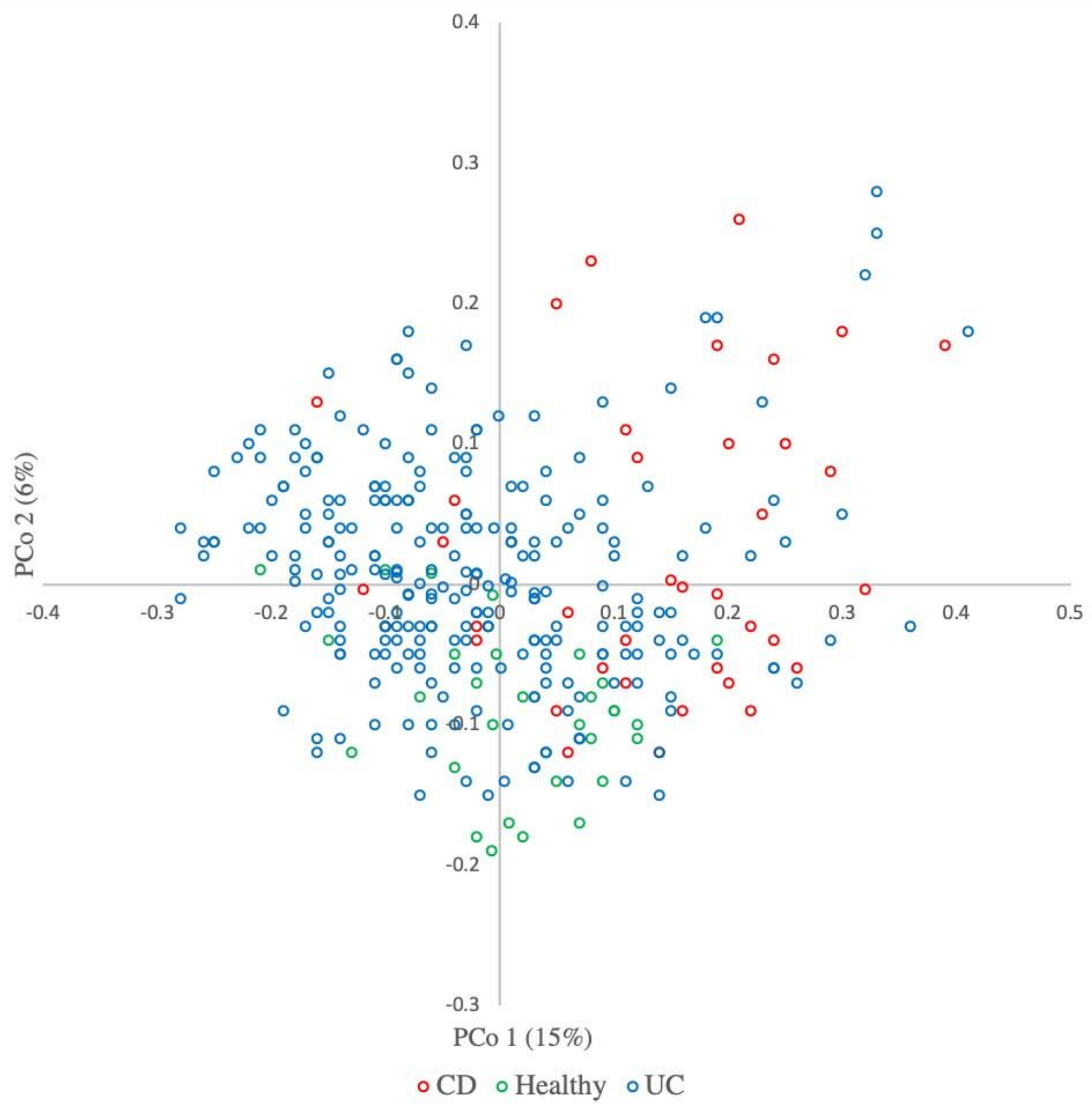

\section{Figure 2}

Principal coordinates analysis plots of beta diversity using unweighted UniFrac distance. CD patients, UC patients and healthy individuals are colored red, blue and green respectively; $p<0.001$, the PERMANOVA. 


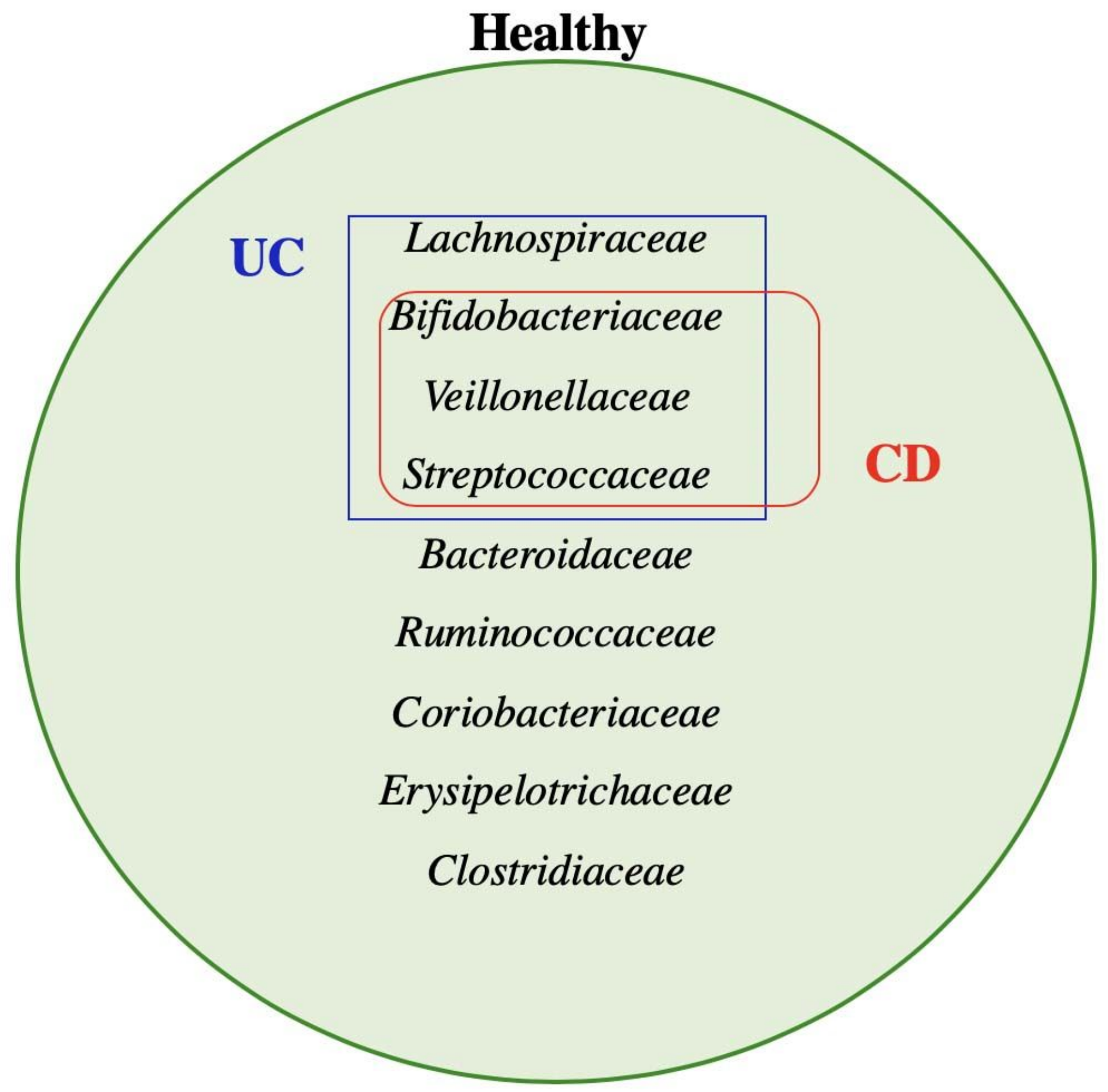

Figure 3

Core gut microbiome of CD patients, UC patients and healthy individuals. 


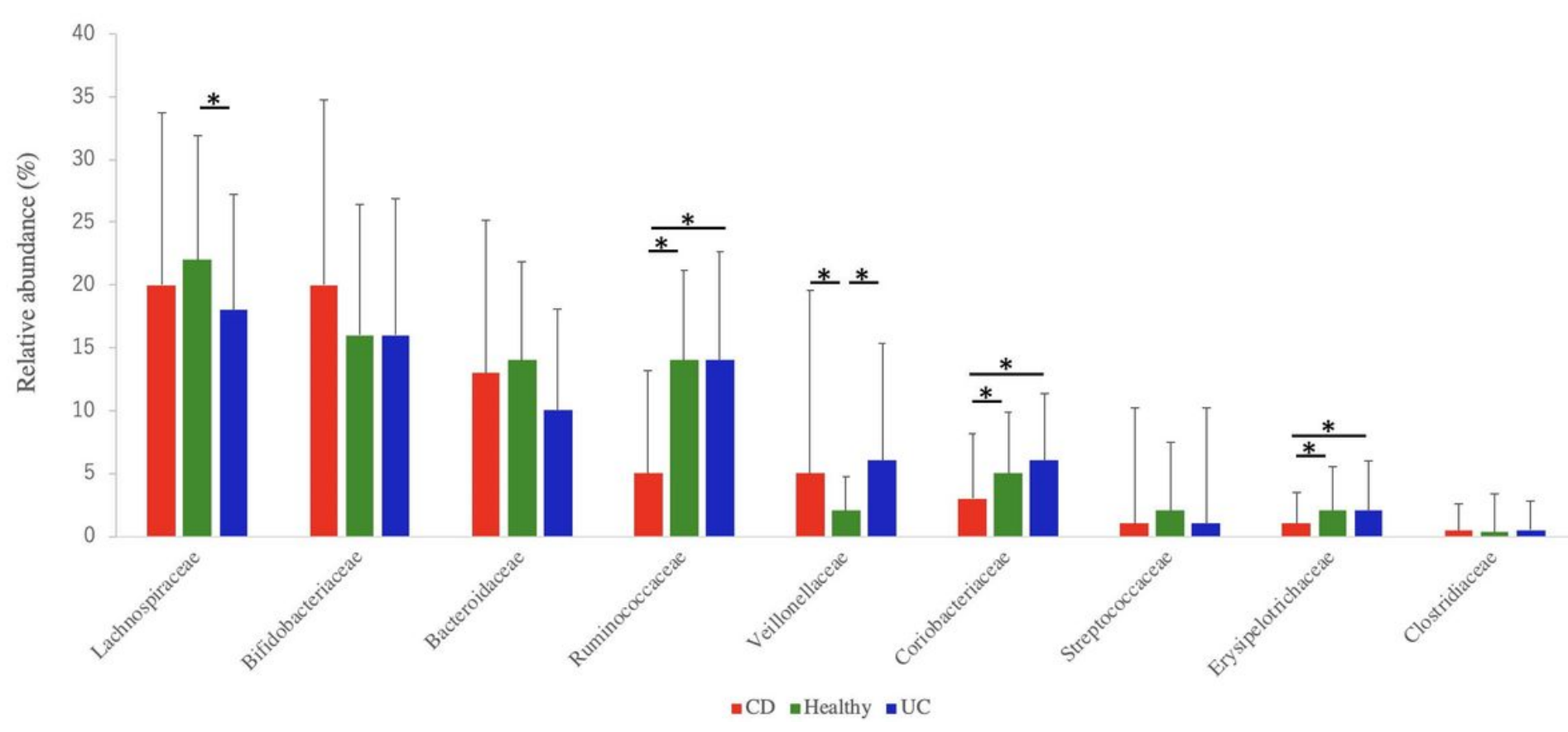

Figure 4

Relative abundances (\%) of core gut bacterial genera in CD patients, UC patients and healthy individuals. ${ }^{*} \mathrm{p}<0.05$, the Kruskal-Wallis test.

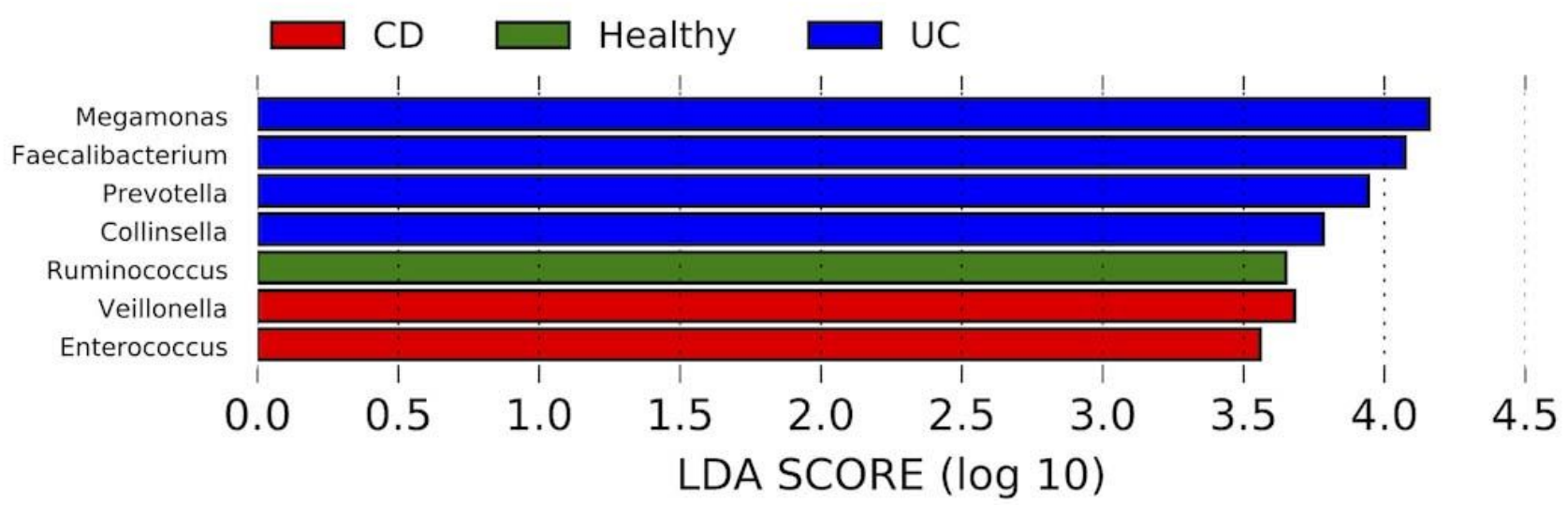

Figure 5

LEfSe represented as a bar plot. Bars represent linear discriminant analysis scores.

\section{Supplementary Files}

This is a list of supplementary files associated with this preprint. Click to download.

- Suppl.Fig1.tiff 
- Suppl.Fig3.tiff

- Suppl.Fig4.tiff

- Suppl.Fig2.tiff

- Suppl.Fig7.tiff

- Suppl.Fig6.tiff

- Suppl.Fig5.tiff 